\title{
DETECÇÃO DE FUNGOS EM SEMENTES DE PAU-BRASIL (Caesalpinia echinata) COLETADAS DURANTE SUA FORMAÇÃO E DISPERSÃO ${ }^{1}$
}

\author{
TATHIANA LISBÔA PADULLA², MARIA HELOISA DUARTE DE MORAES ${ }^{3}$, \\ CLAUDIO JOSÉ BARBEDO ${ }^{4}$, IGOR FERRARI BORGES 5 , JOSÉ OTÁVIO MACHADO MENTEN 6 , \\ SÉRGIO FLORENTINO PASCHOLATI ${ }^{7}$
}

\begin{abstract}
RESUMO - O pau-brasil é uma espécie florestal que tem imenso potencial de utilização, porém, atualmente, encontra-se enquadrada na categoria de extinção sendo importantes as ações que contribuírem para retirá-la desse contexto. As espécies florestais tropicais regeneram-se por vários mecanismos e, dentre estes, a semente é um dos principais, devendo-se atentar para sua qualidade sanitária e fisiológica. Existem poucos relatos sobre a ocorrência de fungos em sementes de paubrasil e os trabalhos existentes não esclarecem em que momento da formação das sementes ocorre a incidência desses fungos. Objetivou-se através deste trabalho verificar a incidência de fungos durante o processo de formação e dispersão das sementes de pau-brasil. Foram analisadas, pelo método do papel de filtro, sementes coletadas em diferentes momentos da sua formação (após antese e após deiscência). Os principais fungos detectados foram Cladosporium cladosporioides, Pestalotiopsis maculans, Fusarium sp. e Epicoccum sp. Outros fungos encontrados em menor incidência foram Aspergillus spp., Nigrospora sp. e Penicillium sp. C. cladosporioides e Pestalotiopsis maculans estavam presentes nas sementes desde o início, na coleta aos 40 dias pósantese, tendo a incidência aumentada ao longo do tempo, atingindo $100 \%$ nas sementes com um e dois dias de deiscência. Fusarium sp. foi detectado nas sementes coletadas após a deiscência, tendo sua incidência aumentada à medida que as sementes permaneceram no solo.
\end{abstract}

Termos para indexação: sanidade, antese, deiscência.

\section{DETECTION OF FUNGI IN BRAZILWOOD SEEDS (Caesalpinia echinata Lam.) COLLECTED DURING SEED FORMATION AND DISPERSION}

\begin{abstract}
Brazilwood, also known as Pau-Brasil or Pernambuco, (Caesalpinia echinata Lam. Syn. Guilandina echinata (Lam.) Spreng.) is a Brazilian forest tree species which has important commercial uses but is at present listed as an endangered species by the International Union for Conservation of Nature (IUCN). Removal of brazilwood from the IUCN endangered species
\end{abstract}

${ }^{1}$ Submetido em 20/08/2009. Aceito para publicação em 08/12/2009. Parte da dissertação de Mestrado do primeiro autor apresentada a ESALQ/ USP.

${ }^{2}$ Bióloga, pós-graduanda do Depto. de Microbiologia Agrícola, USP/ ESALQ, talispadulla@gmail.com.br.

${ }^{3}$ Eng. Agr., Dr., Depto Fitopatologia e Nematologia, ESALQ/USP, mhdmorae@esalq.usp.br.

${ }^{4}$ Eng. Agr., Dr , Instituto de Botânica, Av. Miguel Stéfano 3687, 04301-012
São Paulo, SP, claudio.barbedo@pesquisador.cnpq.br.

${ }^{5}$ Biólogo, M.Sc., BASF S.A. Produtos Agrícolas, Estrada Samuel Aizemberg 1707, São Bernardo do Campo, SP. igor.borges@basf.com.

${ }^{6}$ Eng. Agr., Dr., Professor, Depto. de Fitopatologia e Nematologia, ESALQ/ USP, CP 9, CEP:13418-900. Piracicaba, jomenten@esalq.usp.br.

${ }^{7}$ Biólogo, Dr., Professor, Depto. de Fitopatologia e Nematologia, ESALQ/ USP, CP 9, Piracicaba, sfpascho@esalq.usp.br. 
list is important roe its continued comercial utilization. Tropical forest species are propagated by several mechanisms but seeds are one of the main processes, which means that the health and physiological quality of the seeds of such species are important factors in their propagation. Our objective was to verify the incidence of fungi during the formation and dispersion of $C$. Echinata seeds because there are few accounts regarding the incidence of fungi in C. Echinata seeds and the reports that do exist do not clarify the point in seed formation when fungal infection occurs. We used the health-test to analyze seeds collected at different seed formation stages (post-anthesis and dehiscence). The main fungi detected being Cladosporium cladosporioides, Pestalotiopsis maculans and Epicoccum sp, with a lower incidence of Aspergillus sp, Fusarium sp., Nigrospora sp. and Penicillium sp. We found that $C$. cladosporioides and P.maculans were present in seeds since the beginning of seed formation, at the 40 days post-anthesis collection, and that the incidence of fungi increased with time reaching $100 \%$ in seeds with one and two days of dehiscence. We detected Fusarium sp. in seeds collected after dehiscence and the incidence increased while the seeds remained in contact with soil.

Index terms: anthesis, dehiscence, health.

\section{INTRODUÇÃO}

O pau-brasil (Caesalpinia echinata, Lam; Leguminosae-Caesalpiniodeae) é uma espécie florestal que tem imenso potencial de utilização, podendo-se destacar a arborização urbana e a fabricação de instrumentos musicais (Aguiar e Barbosa, 1985; Ramalho, 1978; Soares, 1985). Atualmente essa espécie encontra-se enquadrada na categoria de extinção, conforme a Portaria IBAMA n. 37-N de 03 de abril de 1992 (Ibama, 1992), sendo importantes as ações que contribuírem para retirá-la dessa lista.

As espécies florestais tropicais regeneram-se por vários mecanismos e, dentre estes, a semente é um dos principais, podendo ser sementes recém-dispersadas ou sementes dormentes no solo (Garwood, 1989). A produção, obtenção e manutenção de sementes com elevada qualidade assumem papel fundamental na preservação das espécies, devendose destacar a grande importância da qualidade sanitária. Microrganismos podem se associar às sementes causando danos na qualidade fisiológica, como a queda no vigor e/ ou na porcentagem de germinação das sementes, e também transmitindo doenças que podem afetar sua produtividade (Carvalho e Nakagawa, 1980; Machado, 1988).

Vários autores, avaliando sementes de diferentes espécies florestais, constataram a incidência de fungos como Alternaria alternata, Aspergillus spp., Cladosporium sp., Curvularia sp., Fusarium spp., Pestalotiopsis sp., Phomopsis sp., Rhizopus sp., Trichoderma sp., entre outros (Cherobini et al., 2004; Grandis et al., 2004; Nascimento et al., 2004; Rego et al., 2004; Santos et al., 2001; Santos et al., 2000). Para sementes de pau-brasil o número de trabalhos é escasso;
Mendes et al. (2004) observaram a presença de Pestalotiopsis sp., Nigrospora sp., Curvularia sp., Fusarium sp. e Aspergillus niger. Lisbôa et al. (2004), relataram a presença de Pestalotiopsis sp. e Cladosporium cladosporioides em alta incidência em sementes de pau-brasil que apresentaram baixo potencial germinativo e número elevado de sementes mortas.

Os poucos trabalhos existentes não esclarecem em que momento da formação das sementes ocorre a incidência desses fungos. Segundo Menten e Bueno (1987), o primórdio da semente pode ser infectado diretamente por agentes infectantes da planta-mãe ou por agentes externos. Pela planta-mãe, a infecção pode ocorrer através do pedúnculo de flores e frutos e do pedúnculo da semente, ou pela superfície íntegra da semente. O inóculo vindo de fonte externa pode atingir flores, frutos e sementes indiferenciadas ou frutos e sementes maduros, podendo ter sido originado em outras plantas, em outro local de infecção da planta-mãe ou no solo.

Objetivou-se através deste trabalho verificar a incidência de fungos durante o processo de formação e dispersão das sementes de pau-brasil.

\section{MATERIAL E MÉTODOS}

O presente trabalho foi desenvolvido no laboratório de Patologia de Sementes do Depto. de Fitopatologia e Nematologia da Escola Superior de Agricultura "Luiz de Queiroz"/USP.

Assementes depau-brasil foram coletadas em diferentes momentos de seu desenvolvimento e após a deiscência, na 
Reserva Biológica de Mogi-Guaçu. Primeiramente foram coletados os frutos com 40, 50 e 60 dias pós-antese, em 30/09, $10 / 10$ e 20/10/2002. A seguir, coletaram-se as sementes com um, dois, três e mais de três dias de deiscência. Os frutos coletados foram levados ao laboratório, as sementes retiradas, sob condições assépticas, e submetidas ao teste de sanidade pelo método do papel de filtro (Lucca Filho, 1987). Esse teste consistiu em colocar, em caixas do tipo gerbox, duas folhas de papel tipo Germitest, previamente umedecidas até a saturação com água destilada e, sobre estas, 20 sementes dispostas de maneira eqüidistantes. A seguir, as caixas foram colocadas em câmara de incubação sob temperatura de $20 \pm 2{ }^{\circ} \mathrm{C}$ e luz alternada ( $12 \mathrm{~h}$ de luz branca fluorescente $/ 12 \mathrm{~h}$ de escuro), durante sete dias. A micota desenvolvida foi detectada e identificada sob microscópio estereoscópico, observandose as estruturas dos fungos (Barnet e Hunter, 1998). Alguns isolados fúngicos foram enviados ao Instituto de Botânica de São Paulo, para serem identificados pela pesquisadora Dra. Rosely A. P. Grandi. As sementes coletadas após a deiscência também foram submetidas ao teste de sanidade.

O delineamento experimental utilizado foi inteiramente casualizado, com cinco repetições de 20 sementes de cada amostra para o teste de sanidade. Os dados foram submetidos à análise de variância e os resultados comparados pelo teste de Tukey a 5\% de probabilidade.

\section{RESULTADOS E DISCUSSÃO}

Pode-se constatar pelos resultados encontrados na Tabela 1, que os fungos Pestalotiopsis maculans e Cladosporium cladosporioides (Hutchison e Reid, 1988) estavam presentes desde o início da formação das sementes, na coleta aos 40 dias pós-antese, tendo a incidência aumentada com o passar do tempo, atingindo $100 \%$ nas sementes com um e dois dias de deiscência. Observou-se menor incidência nas sementes com mais de três dias de deiscência, sendo mais acentuada para $C$. cladosporioides.

\section{TABELA 1. Incidência de fungos em sementes de pau-brasil coletadas durante o processo de formação e deiscência das sementes.}

Incidência (\%) de fungos

\begin{tabular}{ccccccc}
\hline Épocas de Coleta & Pestalotiopsis maculans & Cladosporium cladosporioides & Epicoccum sp. & Penicillium sp. & Fusarium sp. \\
\hline 40 dias pós-antese & $15 \mathrm{c}^{*}$ & $8 \mathrm{~d}$ & $0 \mathrm{c}$ & $0 \mathrm{~d}$ & $0 \mathrm{~d}$ \\
50 dias pós-antese & $12 \mathrm{c}$ & $51 \mathrm{c}$ & $0 \mathrm{c}$ & $0 \mathrm{~d}$ & $0 \mathrm{~d}$ \\
60 dias pós-antese & $85 \mathrm{~b}$ & $91 \mathrm{~b}$ & $30 \mathrm{a}$ & $3 \mathrm{~cd}$ & $0 \mathrm{~d}$ & $8 \mathrm{~cd}$ \\
1 dia de deiscência & $100 \mathrm{a}$ & $100 \mathrm{a}$ & $19 \mathrm{ab}$ & $30 \mathrm{a}$ & $8 \mathrm{~cd}$ \\
2 dias de deiscência & $100 \mathrm{a}$ & $100 \mathrm{a}$ & $12 \mathrm{ab}$ & $8 \mathrm{bc}$ & $14 \mathrm{bc}$ \\
3 dias de deiscência & $98 \mathrm{ab}$ & $98 \mathrm{ab}$ & $10 \mathrm{~b}$ & $20 \mathrm{ab}$ & $34 \mathrm{~b}$ \\
Mais dias de deiscência & $81 \mathrm{~b}$ & $19 \mathrm{~d}$ & $12 \mathrm{ab}$ & $0 \mathrm{~d}$ & $80 \mathrm{a}$ \\
\hline
\end{tabular}

*Médias seguidas por letras distintas, nas colunas, diferem entre si pelo teste de Tukey $(0.05 \%)$

Borges et al. (2005) constataram que sementes de paubrasil atingiram a maturidade fisiológica por volta de 60-65 dias após antese, quando as sementes tinham 30-40\% de água. Segundo Carvalho e Nakagawa (1980) e Marcos Filho (2005), após o ponto de maturação fisiológica, o teor de água das sementes decresce muito ou pouco, dependendo das condições do ambiente. Sendo Pestalotiopsis maculans e Cladosporium cladosporioides considerados fungos de campo (Neergaard, 1979), a queda no teor de água pode explicar sua diminuição, pois, segundo Wetzel (1987), o desenvolvimento dos fungos de campo requer alta umidade.

$\mathrm{O}$ fato de as sementes de pau-brasil terem permanecido por mais tempo no solo possibilitou que, com três dias de deiscência, entrassem em contato com um número maior de microrganismos. A detecção de Fusarium sp. em sementes após seu contato com o solo, chama a atenção para esse fato. À medida que as sementes permaneceram no solo, sua incidência foi aumentando, atingindo o máximo em sementes com mais de três dias de deiscência o que não era esperado, pois espécies de Fusarium são consideradas fungos de campo, isto é, podem infectar as sementes no período de crescimento e maturação (Wetzel, 1987). Esse resultado demonstra que o inóculo originou-se de fonte externa; segundo Menten e Bueno (1987), o inóculo externo pode vir, também, do solo. 
Villela e Peres (2004), observaram que, quando as sementes são coletadas após queda ao solo, ficam expostas a flutuações de temperatura, umidade relativa, orvalho, chuva, entre outros fatores, podendo favorecer ou não o desenvolvimento de fungos de campo.

A diminuição acentuada de Pestalotiopsis maculans e de C. cladosporioides, apesar de ainda terem permanecido em contato com o solo, pode ser explicada pelo aumento na incidência de Fusarium sp., podendo ter ocorrido uma competição entre esses microrganismos.

Dentre os fungos detectados, Pestalotiopsis maculans é, potencialmente, o mais importante pelos danos relatados. Segundo Dhingra et al. (2002), este fungo foi encontrado em associação com sementes de outras espécies florestais como Anadenanthera macrocarpa (Angico Vermelho), sendo altamente patogênico para as sementes e as plântulas, causando podridão na semente, podridão de raiz, redução na altura e definhamento de plântulas sobreviventes. Mendes e Muchovej (1991) relataram a presença de Pestalotiopsis maculans em folhas de pau-brasil, causando lesões claras, variando para marrom, no meio ou no bordo do folíolo. Santos et al. (2001) citam Pestalotiopsis sp. como agente causal de podridões de sementes em condições inadequadas de armazenamento.

Cladosporium cladosporioides tem sido detectado com frequência e em alta incidência em sementes de espécies nativas (Faiad et al., 2004; Lisbôa et al., 2004; Botelho, 2006). Faiad et al. (2004), estudando diversas espécies, constataram a presença de Cladosporium sp., entre outros fungos, em alta frequência; segundo esses autores esse fungo pode ser responsável pela descoloração das sementes e redução na germinação. Dentre os fungos potencialmente patogênicos à espécies florestais, os do gênero Fusarium são responsáveis pelo tombamento tanto de pré como de pós-emergência das plântulas (Carneiro, 1987).

Epicoccum sp. foi observado em baixa porcentagem, a partir de 60 dias pós-antese. Não houve diferença estatística entre sua incidência em sementes coletadas nesse período, excetuando-se para as coletadas aos três dias de deiscência, que diferiu das coletadas aos 60 dias pós-antese, mas não das coletadas nos demais períodos. Penicillium sp. não apresentou comportamento definido, sendo detectado a partir de 60 dias pós-antese, aumentando e diminuindo sucessivamente até não ser detectado na última coleta.

Os resultados deste experimento indicaram que os fungos Pestalotiopsis maculans, C. cladosporioides, Epicoccum sp. e Penicillium sp. podem ter infectado as sementes por inóculo da planta-mãe ou por inóculo externo ainda na árvore, enquanto que a infecção por Fusarium sp. ocorreu por inóculo externo.
Verifica-se que, do ponto de vista sanitário, a melhor época de coleta das sementes é entre 40 e 50 dias pós-antese, pela baixa incidência dos fungos. Porém, Borges et al. (2005) constataram que sementes de pau-brasil atingem a maturidade fisiológica apenas aos $60-65$ dias pósantese, fazendo-se necessários estudos que indiquem uma maneira de controlar a ocorrência dos fungos antes dessa fase para que sementes, na maturidade fisiológica, tenham boa qualidade sanitária.

\section{CONCLUSÕES}

Os fungos Pestalotiopsis maculans, Cladosporium cladosporioides, Epicoccum sp. e Penicillium sp. podem infectar as sementes por inóculo da planta-mãe ou por inóculo externo ainda na árvore de pau-brasil.

A infecção das sementes por Fusarium sp. ocorre por inóculo externo.

Sementes coletadas entre 40 e 50 dias pós-antese possuem qualidade sanitária superior.

\section{AGRADECIMENTOS}

Os autores agradecem à Reserva Biológica de MogiGuaçu, à Reserva de Tapacurá em Pernambuco, ao Jardim Botânico de São Paulo, pelo envio das amostras, à Dra. Rosely Grandi, do Instituto de Botânica, pela identificação da espécie do fungo Cladosporium, à FAPESP pelo auxílio financeiro ao projeto (Proc. 00/06422-4).

\section{REFERÊNCIAS}

AGUIAR, F.F.A.; BARBOSA, J.M. Estudo de conservação e longevidade de sementes de pau-brasil (Caesalpinia echinata Lam.). Ecossistema, v.10, p.145-50,1985.

BARNETT, H.L.; HUNTER, B.B.; Illustrated Genera of Imperfect Fungi. St. Paul: The American Phytopathological Society. 1998. 218p.

BORGES, I.F.; GIUDICE NETO, J.D.; BILIA, D.A.C.; FIGUEIREDO-RIBEIRO, R.C.L.; BARBEDO, C.J.. Maturation of seeds of Caesalpinia echinata Lam. (Brazilwood), an endangered leguminous tree from the Brazilian Atlantic Forest. Brazilian Archives of Biology and Technology. v.48, n.6, p.851-861, nov. 2005.

BOTELHO, L.S. Fungos associados às sementes de ipê-amarelo (Tabebuia serratifolia), ipê-roxo (Tabebuia impetiginosa), aroeira-pimenteira (Schinus terebinthifolius) e aroeira-salsa (Schinus molle): incidência, efeitos na germinação, transmissão para plântulas e controle. 
2006. 114f. Dissertação (Mestrado em Microbiologia Agrícola). Escola Superior de Agricultura Luiz de Queiroz, Piracicaba, 2006.

CARNEIRO, J.S. Testes de sanidade de sementes de essências florestais. In: SOAVE, J.; WETZEL, M.M.V. DA S. (Ed.) Patologia de Sementes. Campinas: Fundação Cargill, cap.17, p.386-394. 1987.

CARVALHO, N.M.; NAKAGAWA, J. Sementes: ciência, tecnologia e produção. Campinas: Fundação Cargill, 1980. $326 \mathrm{p}$.

CHEROBINI, E.A.I.; MUNIZ, M.F.B.; HOPPE, J.M.; ÁVILA, A.L.; CAMARGO, R.F. Qualidade sanitária de sementes de Eugenia involucrata DC, Eugenia pyriformis Cambessedes, Feijoa sellowiana Berg, Psidium cattleianum Sabine. In: SIMPÓSIO BRASILEIRO DE PATOLOGIA DE SEMENTES, 8., 2004, João Pessoa. Palestras e Resumos: João Pessoa, 2004. p.163.

DHINGRA, O.D.; MAIA, C.B.; MESQUITA, J.B. Seedborme pathogenic fungi that affect seedling quality of red angico (Anadenanthera macrocarpa) trees in Brazil. Phytopathology, v.150, p.451-55. 2002.

FAIAD, M.G.R.; RAMOS, V.R.; WETZEL, M.M.V. Patologia de espécies florestais do cerrado. In: SIMPÓSIO BRASILEIRO DE PATOLOGIA DE SEMENTES, 8, 2004, João Pessoa. Palestras e Resumos: João Pessoa, 2004. p.3642.

GARWOOD, N.C. Tropical soil seed banks: a review. In: LECK, M.A.; PARKER, V.T.; SIMPSON, R.L. (Ed.). Ecology of soil seed banks. San Diego: Academic Press. 1989. p.149-209.

GRANDIS, A.; GODOI, S.; MORAES, M.H.D.; MENEGHETTI, C.S.B. Qualidade sanitária das sementes de Astronium graveolens (Guaritá). In: SIMPÓSIO BRASILEIRO DE PATOLOGIA DE SEMENTES, 8, 2004, João Pessoa. Palestras e Resumos: João Pessoa, 2004. p.177.

HUTCHISON, L.J.; REID, J. Taxonomy of some potential wood-staining fungi from New Zeland (2. Pyrenomycetes, Coelomyctes and Hyphomycetes). New Zeland Journal of Botany, v.26, p.83-98, 1988.

IBAMA. Lista oficial de espécies da flora brasileira ameaçadas de extinção. (unpublished). 4pp. Sociedade Botânica do Brasil. Centúria plantarum Brasiliensium extintions minitata. Sociedade Botânica do Brasil. 1992. p.175.

LISBÔA, T.; MORAES, M.H.D.; MENTEN, J.O.M. Sanidade e potencial germinativo de sementes de Caesalpinia echinata, Lam (pau-brasil) coletadas no campus da ESALQ/USP, em
Piracicaba. In: SIMPÓSIO BRASILEIRO DE PATOLOGIA DE SEMENTES, 8, 2004, João Pessoa. Palestras e Resumos: João Pessoa, 2004. p.182.

LUCCA FILHO, O.A. Metodologia dos testes de sanidade de sementes. In: SOAVE, J.; WETZEL, M.M.V. DA S. (Ed.) Patologia de sementes. Campinas: Fundação Cargill, 1987. p.276-298.

MACHADO, J.C. Patologia de sementes: fundamentos e aplicações. Brasília, DF: MEC/ESAL/FAEPE, 1988. 106p.

MARCOS FILHO, J. Fisiologia de sementes de plantas cultivadas. Piracicaba: FEALQ, 2005. 495p.

MENDES, M.L.; MUCHOVEJ, J.J. Pestalotiopsis leaf spot of brazil Wood, Caesalpinia echinata. Plant Pathology, v.40, p.635-636, 1991.

MENDES, S.S.; SANTOS, D.M.; MOURA, A.O.; FRANCO FILHO, E.; MESQUITA, J.B. Qualidade sanitária de sementes de pau-brasil (Caesalpinia echinata Lam.). In: SIMPÓSIO BRASILEIRO DE PATOLOGIA DE SEMENTES, 8, 2004, João Pessoa. Palestras e Resumos: João Pessoa, 2004. p.193.

MENTEN, J.O.M.; BUENO, J.T. Transmissão de patógenos pelas sementes. In: SOAVE, J.; WETZEL, M.M.V. DA S. (Ed.). Patologia de sementes. Campinas: Fundação Cargill, 1987. p.164-191.

NASCIMENTO, L.C.; NERY, A.R.; ARAÚJO, E.; SOUTO, F.M.; ALVES, E.U.; ALMEIDA, F.A. Incidência de fungos em sementes de espécies nativas do Semi-árido Nordestino. In: SIMPÓSIO BRASILEIRO DE PATOLOGIA DE SEMENTES, 8., 2004, João Pessoa. Palestras e Resumos: João Pessoa, 2004. p.197.

NEERGaARD, P. Seed Pathology. London: Mac Millan Press, 1979. v.1, 839p.

RAMALHO, R.S. Pau-brasil (Caesalpinia echinata Lam.). Viçosa, MG: Universidade Federal de Viçosa, 1978. 11p. (Boletim de extensão, 12).

REGO, S.S; SANTOS, A.F.; MEDEIROS, A.C.S.; ABREU, D.C.A. Associação de fungos com frutos e sementes de imbuia (Ocotea porosa). In: SIMPÓSIO BRASILEIRO DE PATOLOGIA DE SEMENTES, 8., 2004, João Pessoa. Palestras e Resumos: João Pessoa, 2004. p.214.

SANTOS, A.F.; GRIGOLETTI JÚNIOR, A.; AUER, C.G. Transmissão de fungos por sementes de espécies florestais. Floresta, v.30, n.1/2, p.119-128, 2000.

SANTOS, A.F.; MEDEIROS, A.C.S.; SANTANA, D.L.Q. Fungos em sementes de espécies arbóreas da mata Atlântica. Colombo, Boletim de Pesquisa Florestal, n.42, p.51-60, jan/jun 2001. 
SOARES, C.M.C. Pau-brasil: a árvore nacional. 2.ed. Recife: Universidade Federal Rural de Pernambuco/Estação Ecológica de Tapacurá. 1985. 39p.

VILLELA, F.A.; PERES, W.B. Coleta, Beneficiamento e Armazenamento. In: FERREIRA, A.G.; BORGHETTI, F.
(Ed.). Germinação - do básico ao aplicado. Porto Alegre: Artmed, 2004. cap. 17, p.266-281.

WETZEL, M. M.V.S. Fungos de armazenamento. In: SOAVE, J.; WETZEL, M.M.V.S. (Ed.) Patologia de sementes. Campinas: Fundação Cargill, 1987. p.260-275. 